\title{
The role of test trials in paired-associate learning
}

GREGORY L. BELENKY, STANFORD MEDICAL SCHOOL MICHAEL COLE, UNIVERSITY OF CALIFORNIA, IRVINE

This study investigated the effect of varying the events occurring between two reinforced trials on the course of learning. It was found that the effectiveness of a second reinforced trial was greater; (a) for the longer of two inter-reinforcement intervals prior to the second reinforced trial; (b) if $S$ is not specifically tested for retention of the reinforced item; (c) for filler trials that made no specific demands on $S$.

A general issue raised by the studies concerning the "all or none versus incremental" nature of paired associate verbal learning concerns the role of test trials.

Izawa (1966a) showed that although no learning of correct responses occurs on consecutive test trials, test trials do increase the effectiveness of a following reinforced trial. However, whether this potentiation was a unique result of the testing items per se, or whether it stemmed from the increased temporal spacing of reinforced trials which accompanied the interspersed test trials could not be determined from Izawa's experimental design.

The purpose of the present experiment was to study the effects of different intervals between reinforced trials as a function of the type of event filling these intervals. The experimental design included two intervals between reinforced trials, and three types of events to fill these intervals.

\section{Method}

Subjects and procedure. The Ss were 48 college undergraduates. They were run individually.

The paired associates were presented by means of a Kodak Carousel projector. The presentation interval for all items, whether reinforced presentations (stimulus term paired with response term), test presentations (stimulus term alone), or other filler events (matrices or blanks), was $2 \mathrm{sec}$. There was a .5 sec interval between presentations.

Each S was presented 10 lists of eight paired associates. Guesses were required if $S$ did not know the correct response on a test trial. When a matrix of random numbers appeared, $S$ was required to read off the numbers in the matrix as fast as possible. There were five different matrix slides used for all Ss. The matrices contained more than enough numbers to keep $S$ occupied during the presentation interval. No specific instructions were given $S$ as to what to do during the blank slide presentations.

Design. The experimental design involved the four within-list conditions and the two between-list conditions seen in Table 1. The four within-list conditions which specified reinforcement-test sequence were RTRTT, R-RTT, RTTRTT, and R--RTT. Those items in which there is one event intervening between the two
Table 1: Complete experimental paradigm (including Blank and Matrix conditions).

Cycles through the list: $\begin{array}{llllll}1 & 2 & 3 & 4 & 5 & 6\end{array}$

\begin{tabular}{|c|c|c|c|c|c|c|c|}
\hline \multirow{5}{*}{ Blank } & \multirow{2}{*}{ Short } & Test & (1) & $\mathbf{R} \mathbf{T} \mathbf{R}$ & $\mathrm{T}$ & $\mathbf{T}$ & B \\
\hline & & No Test & (2) & R B R & $T$ & $\mathbf{T}$ & B \\
\hline & \multirow{3}{*}{ Long } & Test & (3) & R T T T & $\mathbf{R}$ & $\mathrm{T}$ & $\mathbf{T}$ \\
\hline & & & & & & & \\
\hline & & No Test & (4) & R B B & $\mathbf{R}$ & $\mathbf{T}$ & $\mathbf{T}$ \\
\hline \multirow{4}{*}{ Matrix } & \multirow{2}{*}{ Short } & Test & (1) & $\mathbf{R} \mathbf{T} \mathbf{R}$ & $\mathrm{T}$ & $\mathrm{T}$ & M \\
\hline & & No Test & (2) & R M R & $\mathrm{T}$ & $\mathrm{T}$ & M \\
\hline & \multirow{2}{*}{ Long } & Test & (3) & R T T & $\mathbf{R}$ & $\mathrm{T}$ & $\mathbf{T}$ \\
\hline & & No Test & (4) & R M M & $\mathbf{R}$ & $\mathbf{T}$ & $T$ \\
\hline
\end{tabular}

(B) stands for a blank slide.

(M) stands for a number matrix.

reinforced trials will be referred to as the "Short" condition and those items in which there are two events intervening will be referred to as the "Long" condition. In a like manner, we will designate items with test trials in the interval between the two reinforced trials as the "Test" condition, and those items without test trials between the two reinforced trials the "No Test" condition.

Within each list of eight items, two items were assigned to each of the four reinforcement-test sequences. In half of the lists presented to each $\mathrm{S}$, the intervals with no test trials were filled with number matrices, and for the other half, the intervals with no test trials were filled with blank slides, thus generating the two between-list conditions. These betweenlist conditions we will refer to as the "Matrix" and "Blank" conditions, respectively.

Two test trials were included following the second reinforced trial in order to obtain data on the changes in proportion correct over consecutive test trials.

The 80 paired associate items used in the experiment consisted of common four and five letter nouns as stimulus terms and two digit numbers as response terms.

The order of sequence within lists and between lists was balanced across all $48 \mathrm{Ss}^{2}$

\section{Results and Discussion}

The mean of correct responses on the test trials following the second reinforced trial are presented in Table 2.

Of primary interest was the effect of the different ways of filling the interval between the two reinforced trials on subsequent test performance. The following conclusions relevant to this question are supported 
Table 2. Mean correct out of $\mathbf{1 0}$ for the first and second test trial following the second reinforced trial for the various experimental conditions.

\begin{tabular}{|c|c|c|c|c|c|c|c|c|}
\hline & & & & $\mathrm{T}_{1}$ & $\mathbf{T}_{2}$ & & $\mathrm{~T}_{1}$ & $\mathrm{~T}_{2}$ \\
\hline \multirow{4}{*}{ Blank } & \multirow{4}{*}{5.948} & \multirow{4}{*}{5.906} & \multirow{2}{*}{ Short } & \multirow{2}{*}{5.834} & \multirow{2}{*}{5.896} & Test & 5.813 & 5.813 \\
\hline & & & & & & No Test & 5.854 & 5.979 \\
\hline & & & \multirow[t]{2}{*}{ Long } & \multirow[t]{2}{*}{6.062} & \multirow[t]{2}{*}{5.916} & Test & 6.042 & 5.833 \\
\hline & & & & & & No Test & 6.083 & 6.000 \\
\hline & $\mathrm{T}_{1}$ & $\mathrm{~T}_{2}$ & & $\mathrm{~T}_{1}$ & $\mathrm{~T}_{2}$ & & $T_{1}$ & $\mathrm{~T}_{2}$ \\
\hline \multirow{4}{*}{ Matrix } & \multirow{4}{*}{5.583} & \multirow{4}{*}{5.500} & Short & 5.483 & 5.312 & Test & 5.458 & 5.104 \\
\hline & & & & & & No Test & 5.417 & 5.521 \\
\hline & & & Long & 5.729 & 5.688 & Test & 5.583 & 5.646 \\
\hline & & & & & & No Test & 5.875 & 5.729 \\
\hline
\end{tabular}

by the analysis (numbers in parentheses refer to the mean number correct out of 10 possible): (1) Two intervening events (5.85) produce superior performance to one intervening event (5.62) $(F=13.5, \mathrm{df}=1 / 47, \mathrm{p}<.01)$. (2) Blank events (5.93) produce superior performance to Matrix events (5.54) $(\mathrm{F}=38,1, \mathrm{df}=1 / 47, \mathrm{p}<.01)$. (3) Test events (5.66) are inferior to No Test events (5.81) $(F=5.5, d f=1 / 47, p<.05)$. (4) No interaction terms involving these variables were significant.

The difference between Test $1(5.76)$ and Test 2 (5.70) following the second reinforced trial was not statistically reliable $(F \simeq 1)$.

These results confirm and extend the earlier finding of Izawa (1966a). In the present case, however, it appears that the type of intervening event makes a difference. Both kinds of nontest filler events produced performance superior to testing, but are not equivalent, since a blank interval is more effective than a matrix-filled interval.

It is difficult to say exactly how the filler events exert their effects. The matrices may set up general interference both because they prevent rehearsal and because they contain numbers, which are similar to the response terms in this experiment. Blanks would seem to allow for rehearsal of items. Test trials may actually be specific interference in this experimental situation. By forcing the $S$, in the early stages of learning, to guess on all test trials on which he does not know the correct response, we may cause him to associate the guessed response with the stimulus term of the

Table 3. Joint proportion of correct and incorrect across consecutive test trials following the second reinforced trial.

\begin{tabular}{lllll} 
& CC & CI & IC & II \\
\hline BlankShort-Test & .500 & .077 & .064 & .358 \\
Blank-Short-No Test & .506 & .069 & .085 & .340 \\
Blank-Long-Test & .527 & .060 & .042 & .371 \\
Blank-Long-No Test & .546 & .081 & .071 & .302 \\
\hline Matrix-Short-Test & .448 & .085 & .050 & .417 \\
Matrix-Short-No Test & .483 & .062 & .069 & .385 \\
Matrix-Long-Test & .479 & .067 & .083 & .371 \\
Matrix-Long-No Test & .521 & .067 & .062 & .350 \\
\hline Overall proportions & .501 & .071 & .066 & .362 \\
\hline (CC) is correct-correct & (IC) is incorrect-correct & \\
(CI) is correct-incorrect & (II) is incorrect-incorrect &
\end{tabular}

item in question, as association which interferes with the effect of the next reinforced trial for that item. Izawa (1966a) also considers this possibility.

A very recent paper by Izawa (1966b) indicates that different combinations of test and blank intervals have different effects. It appears that more variation of filler events will be required to pin down the exact relationships involved.

Another interesting aspect of the results is the joint proportion of correct and incorrect across the two test trials after the second reinforced trial. These data are sum-marized in Table 3. From the table it appears that: (1) There is some forgetting (proportion of items going from correct to incorrect is greater than zero). This does not show up in the analysis of variance which tested for changes in total correct over the two test trials, because the proportion of items going from incorrect to correct is also greater than zero. (2) The proportion of items going from correct to incorrect and from incorrect and from incorrect to correct is small. (3) The proportion of items going from correct to incorrect is greater than the proportion of items going from incorrect to correct.

This pattern of results is relevant to the theoretical controversies in the application of mathematical models to paired associate data (Estes, 1960; Postman, 1963). The fact that the proportion of correct-incorrect is greater than the proportion incorrect-correct, and that both of these proportions are greater than zero, rule out the one-element model, which predicts that both proportions are zero, and linear models, which predict that the two proportions would be equal. The stimulus fluctuation model (see Izawa, 1966a) fits these conditional response data at least qualitatively. The fact that the potentiating effect observed in this experiment can also be predicted by the stimulus fluctuation model indicates that an overall quantitative analysis of the data in terms of this model would be useful. However, a longer sequence of trials would be required to make quantitative predictions.

References

ESTES, W. K. Learning theory and the new "mental chemistry." Psychon. Rev., 1960, 67, 207-223.

IZAWA, C. Reinforcement-test sequences in paired-associate learning. Psych. Rep., 1966, 18, 879-919 (a).

IZAWA, C. Role of reinforcement and test in paired-associate learning. Proceed. of the 74th Annu. Conv. Amer. Psychol. Assoc., Los Angeles: 1966 (b).

POSTMAN, L. J. One-trial learning. In C. N. Cofer, \& B. S. Musgrave, (Eds.) Verbal behavior and learning: problems and processes. New York: McGraw-Hill, 1963, 295-321.

THORNDIKE, R. I., \& LORGE, I. The teacher's word book of 30,000 words. New York: Bureau of Publications, Teachers College, Columbia University, 1944.

Notes

1. This work was conducted as partial fulfillment of the first author's Bachelors Degree from Yale University where the second author was a staff member. Thanks are due A. R. Wagner and the Psychology Department, Yale University, for their assistance in completing this research.

2. Details of list construction and counter balancing procedure are available from the first author on request.

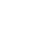

